\title{
ABSENT SPECTRAL GAPS OF THE GENERALIZED KRONIG-PENNEY HAMILTONIANS
}

\author{
Hiroaki NIIKUNI
}

(Received 5 December 2006 and revised 9 April 2007)

\begin{abstract}
We discuss the spectral gaps of the generalized Kronig-Penney Hamiltonians which possess two point interactions in the basic cell $[0,2 \pi)$. We determine whether or not the $j$ th spectral gap of the Hamiltonian is absent for a given $j \in \mathbb{N}$.
\end{abstract}

\section{Introduction and main result}

In this paper we study the spectrum of the one-dimensional Schrödinger operator with particular point interactions on a lattice. The aim of this paper is to determine whether or not the $j$ th gap of its spectrum is absent for a given $j \in \mathbb{N}=\{1,2,3, \ldots\}$.

To describe our main theorem, we introduce the following notation. We fix $\kappa \in(0,2 \pi)$. Let $\Gamma_{1}=2 \pi \mathbb{Z}, \Gamma_{2}=\{\kappa\}+2 \pi \mathbb{Z}, \Gamma=\Gamma_{1} \cup \Gamma_{2}$ and $\tau=2 \pi-\kappa$. Let $\mathrm{SL}_{2}(\mathbb{R})$ be the special linear group, i.e.

$$
\mathrm{SL}_{2}(\mathbb{R})=\left\{\left(\begin{array}{ll}
a & b \\
c & d
\end{array}\right) \mid a, b, c, d \in \mathbb{R}, a d-b c=1\right\} .
$$

Consecutively, we introduce the Sobolev space

$$
H^{2}(I)=\left\{y(x) \in L^{2}(I) \mid y^{\prime}(x), y^{\prime \prime}(x) \in L^{2}(I)\right\},
$$

where the symbol' stands for the differentiation with respect to $x$, and $I \subset \mathbb{R}$ is an open set. For $\theta_{1}, \theta_{2} \in \mathbb{R}$ and $A_{1}, A_{2} \in \mathrm{SL}_{2}(\mathbb{R})$, we define the one-dimensional Schrödinger operator $H=H\left(\theta_{1}, \theta_{2}, A_{1}, A_{2}\right)$ in $L^{2}(\mathbb{R})$ as follows:

$$
\begin{gathered}
(H y)(x)=-y^{\prime \prime}(x), \quad x \in \mathbb{R} \backslash \Gamma, \\
\operatorname{Dom}(H)=\left\{\begin{array}{l}
\left.y \in H^{2}(\mathbb{R} \backslash \Gamma) \mid \begin{array}{c}
y(x+0) \\
y^{\prime}(x+0)
\end{array}\right)=e^{i \theta_{j}} A_{j}\left(\begin{array}{c}
y(x-0) \\
y^{\prime}(x-0)
\end{array}\right) \\
\text { for } x \in \Gamma_{j}, j=1,2
\end{array}\right\} .
\end{gathered}
$$

The operator $H$ is self-adjoint (see [6, Proposition 2.1]). Since $H$ has $2 \pi$-periodic point interactions, we can make use of a direct integral decomposition for $H$ (see [8, Section XIII.16]). For $\mu \in \mathbb{R}$, we define the Hilbert space

$$
\mathcal{H}_{\mu}=\left\{u \in L_{\text {loc }}^{2}(\mathbb{R}) \mid u(x+2 \pi)=e^{i \mu} u(x) \text { for almost every } x \in \mathbb{R}\right\}
$$

2000 Mathematics Subject Classification: Primary 34L15;

Secondary 34B30, 34L05, 34B37.

Keywords and Phrases: generalized point interactions, rotation number, band spectrum, Kronig-Penney Hamiltonians. 
equipped with the inner product

$$
\langle u, v\rangle_{\mathcal{H}_{\mu}}=\int_{0}^{2 \pi} u(x) \overline{v(x)} d x, \quad u, v \in \mathcal{H}_{\mu} .
$$

We define a fiber operator $H_{\mu}=H_{\mu}\left(\theta_{1}, \theta_{2}, A_{1}, A_{2}\right)$ in $\mathcal{H}_{\mu}$ as

$$
\begin{gathered}
\left(H_{\mu} y\right)(x)=-y^{\prime \prime}(x), \quad x \in \mathbb{R} \backslash \Gamma, \\
\operatorname{Dom}\left(H_{\mu}\right)=\left\{\begin{array}{c}
y \in H^{2}((0,2 \pi) \backslash\{\kappa\}), \\
\left.y \in \mathcal{H}_{\mu} \mid \begin{array}{c}
y(x+0) \\
y^{\prime}(x+0)
\end{array}\right)=e^{i \theta_{j}} A_{j}\left(\begin{array}{c}
y(x-0) \\
y^{\prime}(x-0)
\end{array}\right) \\
\text { for } x \in \Gamma_{j}, j=1,2
\end{array}\right\} .
\end{gathered}
$$

We further define a unitary operator $\mathcal{U}$ from $L^{2}(\mathbb{R})$ onto $\int_{0}^{2 \pi} \oplus \mathcal{H}_{\mu} d \mu$ as

$$
(\mathcal{U} u)(x, \mu)=\frac{1}{\sqrt{2 \pi}} \sum_{l=-\infty}^{\infty} e^{i l \mu} u(x-2 l \pi) .
$$

Then we have the direct integral representation of $H$ :

$$
\mathcal{U} H \mathcal{U}^{-1}=\int_{0}^{2 \pi} \oplus H_{\mu} d \mu .
$$

For each $j=1,2$, we write the elements of $A_{j}$ as

$$
A_{j}=\left(\begin{array}{ll}
a_{j} & b_{j} \\
c_{j} & d_{j}
\end{array}\right)
$$

Let $\lambda_{j}(\mu)$ be the $j$ th eigenvalue of $H_{\mu}$ counted with multiplicity for $j \in \mathbb{N}$. Put

$$
\xi= \begin{cases}b_{1} b_{2} & \text { if } b_{1} \neq 0 \neq b_{2}, \\ \left(a_{1}+d_{1}\right) b_{2} & \text { if } b_{1}=0, b_{2} \neq 0 \\ b_{1}\left(a_{2}+d_{2}\right) & \text { if } b_{1} \neq 0, b_{2}=0 \\ \left(a_{1}+d_{1}\right)\left(a_{2}+d_{2}\right) & \text { if } b_{1}=b_{2}=0\end{cases}
$$

Since $A_{j} \in \mathrm{SL}_{2}(\mathbb{R})$, we have $a_{j}+d_{j} \neq 0$ if $b_{j}=0$. This implies that $\xi \neq 0$. Let $\sigma(H)$ be the spectrum of $H$.

To define the spectral gaps of $H$, we now quote the basic properties of $\sigma(H)$ from [7, Proposition 1.1].

PROPOSITION 1.1. We have the following.
(a) The function $\lambda_{j}(\cdot)$ is continuous on $[0,2 \pi]$.
(b) It holds that $\lambda_{j}(\mu)=\lambda_{j}\left(-\mu+2 \theta_{1}+2 \theta_{2}\right)$.
(c) If $\mu-\left(\theta_{1}+\theta_{2}\right) \notin \pi \mathbb{Z}$, then every eigenvalue of $H_{\mu}$ is simple. 
(d) The spectrum of $H\left(\theta_{1}, \theta_{2}, A_{1}, A_{2}\right)$ is given by

$$
\begin{aligned}
\sigma\left(H\left(\theta_{1}, \theta_{2}, A_{1}, A_{2}\right)\right) & =\bigcup_{\mu \in\left[\theta_{1}+\theta_{2}, \theta_{1}+\theta_{2}+\pi\right]} \sigma\left(H_{\mu}\left(\theta_{1}, \theta_{2}, A_{1}, A_{2}\right)\right) \\
& =\bigcup_{j=1}^{\infty} \lambda_{j}\left(\left[\theta_{1}+\theta_{2}, \theta_{1}+\theta_{2}+\pi\right]\right) \\
& =\bigcup_{j=1}^{\infty} \bigcup_{\mu \in\left[\theta_{1}+\theta_{2}, \theta_{1}+\theta_{2}+\pi\right]}\left\{\lambda_{j}(\mu)\right\} .
\end{aligned}
$$

(e) The set $\sigma\left(H\left(\theta_{1}, \theta_{2}, A_{1}, A_{2}\right)\right)$ is independent of $\theta_{1}$ and $\theta_{2}$.

(f) If $\xi>0$ and $\theta_{1}=\theta_{2}=0$, then the function $\lambda_{j}(\cdot)$ is strictly monotone increasing (respectively, decreasing) function on $[0, \pi]$ for odd (respectively, even) $j$.

(g) If $\xi<0$ and $\theta_{1}=\theta_{2}=0$, then the function $\lambda_{j}(\cdot)$ is strictly monotone increasing (respectively, decreasing) function on $[0, \pi]$ for even (respectively, odd) $j$.

Here we define the spectral gaps of $H$. On account of Proposition 1.1(e), we hereafter discuss only the case where

$$
\theta_{1}=\theta_{2}=0
$$

which does not cause any loss of generality. We define

$$
G_{j}= \begin{cases}\left(\lambda_{j}(\pi), \lambda_{j+1}(\pi)\right) & \text { for } j \text { odd }, \\ \left(\lambda_{j}(0), \lambda_{j+1}(0)\right) & \text { for } j \text { even, }\end{cases}
$$

in the case where $\xi>0$, while we put

$$
G_{j}= \begin{cases}\left(\lambda_{j}(\pi), \lambda_{j+1}(\pi)\right) & \text { for } j \text { even, } \\ \left(\lambda_{j}(0), \lambda_{j+1}(0)\right) & \text { for } j \text { odd, }\end{cases}
$$

if $\xi<0$. Then we refer to the open interval $G_{j}$ as the $j$ th gap of the spectrum of $H$. Furthermore, we put $B_{j}=\lambda_{j}([0, \pi])$. This closed interval $B_{j}$ is called the $j$ th band of the spectrum of $H$. As described above, our purpose is to determine whether or not the $j$ th gap is absent for a given $j \in \mathbb{N}$, namely, to clarify the set

$$
\Lambda:=\left\{l \in \mathbb{N} \mid G_{l}=\emptyset\right\} .
$$

Let $E$ be the $2 \times 2$ unit matrix. We are concerned with the case where

$$
A_{1} A_{2}= \pm E \quad \text { and } \quad A_{1}, A_{2} \in \mathrm{SL}_{2}(\mathbb{R}) \backslash\{E,-E\} .
$$

For convenience we rewrite the elements of $A_{1}$ as

$$
A_{1}=\left(\begin{array}{ll}
a & b \\
c & d
\end{array}\right) .
$$

Our main result is the following theorem. 
THEOREM 1.2. Adopt the assumption (1.3). Let $\kappa \neq \pi$.

(a) Assume that $\kappa / \pi \notin \mathbb{Q}$. Then we have

$$
\Lambda= \begin{cases}\{k+1\} & \text { if } d=a, b \neq 0,-c / b=k^{2} / 4 \text { for some } k \in \mathbb{N}, \\ \varnothing & \text { otherwise. }\end{cases}
$$

(b) Suppose that $\kappa / 2 \pi=q / p,(p, q) \in \mathbb{N}^{2}$, and $\operatorname{gcd}(p, q)=1$. Then we have

$$
\Lambda= \begin{cases}\{p j \mid j \in \mathbb{N}\} & \text { if } b=0, \\ \{1+p j \mid j \in \mathbb{N}\} \cup\{1+k\} & \text { if } d=a, b \neq 0,-c / b=k^{2} / 4, \\ & \text { for some } k \in \mathbb{N}, k \neq 0(\bmod p), \\ \{1+p j \mid j \in \mathbb{N}\} & \text { otherwise. }\end{cases}
$$

The Schrödinger operators with periodic point interactions have been discussed in numerous works $[\mathbf{1 - 6 , 9 , 1 0 ]}$. To see the background of our study, we give a review on these works. In 1931, Kronig and Penney [5] first introduced the concept of the Schrödinger operators with periodic singular potentials. They inspired so-called the Kronig-Penney Hamiltonian which is formally expressed as

$$
L_{1}=-\frac{d^{2}}{d x^{2}}+\beta \sum_{l=-\infty}^{\infty} \delta(x-2 \pi l) \quad \text { in } L^{2}(\mathbb{R}),
$$

where $\delta(x)$ is the Dirac delta function at the origin and $\beta \in \mathbb{R} \backslash\{0\}$. The operator $L_{1}$ is the most fundamental Hamiltonian in solid-state physics. The precise definition of this operator is given through boundary conditions on the lattice $2 \pi \mathbb{Z}$ as follows.

$$
\begin{gathered}
\left(L_{1} y\right)(x)=-y^{\prime \prime}(x), \quad x \in \mathbb{R} \backslash 2 \pi \mathbb{Z}, \\
\operatorname{Dom}\left(L_{1}\right)=\left\{\begin{array}{l}
\left.y \in H^{2}(\mathbb{R} \backslash 2 \pi \mathbb{Z}) \mid \begin{array}{c}
y(x+0) \\
y^{\prime}(x+0)
\end{array}\right)=M_{1}\left(\begin{array}{c}
y(x-0) \\
y^{\prime}(x-0)
\end{array}\right) \\
\text { for } x \in 2 \pi \mathbb{Z}
\end{array}\right\},
\end{gathered}
$$

where

$$
M_{1}:=\left(\begin{array}{cc}
1 & 0 \\
\beta & 1
\end{array}\right) .
$$

Namely, the operator $L_{1}$ is expressed as $L_{1}=H\left(0,0, M_{1}, E\right)$ in our notation. Afterwards, Gesztesy, Holden and Kirsch [2, 3] inspired a new class of point interactions. They studied the spectral properties of the operator $L_{2}:=H\left(0,0, M_{2}, E\right)$ in $L^{2}(\mathbb{R})$, where

$$
M_{2}=\left(\begin{array}{cc}
1 & \beta \\
0 & 1
\end{array}\right) \text {. }
$$

This operator has the formal expression

$$
L_{2}=-\frac{d^{2}}{d x^{2}}+\beta \sum_{l=-\infty}^{\infty} \delta^{\prime}(x-2 \pi l) \quad \text { in } L^{2}(\mathbb{R}) .
$$

They proved that the $j$ th gap of $\sigma\left(L_{2}\right)$ is absent if and only if $j=1$ and $\beta=-2 \pi$. They also proved that every gap of $\sigma\left(L_{1}\right)$ is present. In 1986, these two types of point interactions were 
widely generalized by Šeba [9]. He discovered that the domain of any self-adjoint extension of $\left.\left(-d^{2} / d x^{2}\right)\right|_{C_{0}^{\infty}(\mathbb{R} \backslash\{0\})}$ in $L^{2}(\mathbb{R})$ of coupled type is expressed as

$$
\left\{y \in H^{2}(\mathbb{R} \backslash\{0\}) \mid\left(\begin{array}{c}
y(+0) \\
y^{\prime}(+0)
\end{array}\right)=e^{i \theta} A\left(\begin{array}{c}
y(-0) \\
y^{\prime}(-0)
\end{array}\right)\right\}
$$

with $A \in \mathrm{SL}_{2}(\mathbb{R})$ and $\theta \in \mathbb{R}$. Šeba [9] and Chernoff and Hughes [1] proved that the operators in a particular class of the self-adjoint extensions can be approximated by the Schrödinger operators with local short-range potentials in the strong resolvent sense. In [4], Hughes gave the Floquet-Bloch decomposition of the Schrödinger operator with generalized point interactions of the form $L_{3}:=H\left(\theta_{1}, 0, A_{1}, E\right)$, where $\theta_{1} \in \mathbb{R}$ and $A_{1} \in \mathrm{SL}_{2}(\mathbb{R})$. Yoshitomi [10] investigated the spectral gaps of the operators

$$
\begin{aligned}
& P_{0}=-\frac{d^{2}}{d x^{2}}+\sum_{l=-\infty}^{\infty}\left(\beta_{1} \delta(x-\kappa-2 \pi l)+\beta_{2} \delta(x-2 \pi l)\right) \text { in } L^{2}(\mathbb{R}), \\
& P_{1}=-\frac{d^{2}}{d x^{2}}+\sum_{l=-\infty}^{\infty}\left(\beta_{1} \delta^{\prime}(x-\kappa-2 \pi l)+\beta_{2} \delta^{\prime}(x-2 \pi l)\right) \text { in } L^{2}(\mathbb{R}) .
\end{aligned}
$$

For $j \in \mathbb{N}$ and $k \in\{0,1\}$, he proved that $\sigma\left(P_{k}\right)$ has an absent gap if and only if

$$
\beta_{1}+\beta_{2}=0
$$

and $\kappa / \pi \in \mathbb{Q}$ hold. He also showed that if $\beta_{1}+\beta_{2}=0$ and $\kappa / 2 \pi=m / n,(m, n) \in \mathbb{N}^{2}$, and $\operatorname{gcd}(m, n)=1$, then the $j$ th gap of $\sigma\left(P_{k}\right)$ is absent if and only if $j-k \in n \mathbb{N}$.

These backgrounds attract our interest to the generalized Kronig-Penney Hamiltonians $H=H\left(\theta_{1}, \theta_{2}, A_{1}, A_{2}\right)$ defined in (1.1) and (1.2). However, since this operator involves 10 real parameters $\theta_{1}, \theta_{2}, a_{1}, b_{1}, c_{1}, d_{1}, a_{2}, b_{2}, c_{2}$ and $d_{2}$, the spectral gaps are more or less complicated to analyze. To eliminate the complication, we assumed that $A_{1}, A_{2} \in \mathrm{SO}(2) \backslash$ $\{E,-E\}$ in our previous study [6]. Under this assumption, we may write the elements of $A_{j}$ as

$$
A_{j}=\left(\begin{array}{cc}
\cos \alpha_{j} & -\sin \alpha_{j} \\
\sin \alpha_{j} & \cos \alpha_{j}
\end{array}\right) \quad \text { and } \quad \alpha_{j} \in(-\pi, 0) \cup(0, \pi) .
$$

We assume that

$$
\alpha_{1}+\alpha_{2} \equiv 0 \quad(\bmod \pi)
$$

and $\kappa \neq \pi$. Then the following claims hold true; see [6, Theorem 1.3].

(i) Let $\kappa / \pi \notin \mathbb{Q}$. Then we have $G_{j}=\emptyset$ if and only if $j=3$.

(ii) If $\kappa / 2 \pi=q / p,(p, q) \in \mathbb{N}^{2}$, and $\operatorname{gcd}(p, q)=1$, then

$$
\Lambda=\{3\} \cup\{p k+1 \mid k \in \mathbb{N}\} .
$$

This is the first example of the existence of the absent spectral gaps in the case where $\kappa / \pi \notin \mathbb{Q}$. Our assumption (1.3) in Theorem 1.2 includes the case (1.5) or (1.6). In this sense, our Theorem 1.2 is a generalization of the results in $[\mathbf{6}, \mathbf{1 0}]$.

This paper is organized as follows. In Section 2, we locate the absent gaps of $\sigma(H)$, namely, we evaluate the set $\bigcup_{k=1}^{\infty} B_{k} \cap B_{k+1}$ in an explicit way. To this end we utilize the monodromy matrix $(2.5)$. In a similar way to $[\mathbf{6}, \mathbf{1 0}]$, we reduce the problem to a system 
of algebraic equations. However, since we deal with a more general case than in $[\mathbf{6}, \mathbf{1 0}]$, we must execute rather harder tasks than in $[6,10]$. In Section 3 , we quote our previous result [7] which describes the properties of the rotation number for the generalized KronigPenney Hamiltonians. The rotation number plays an important role in this paper. To determine the indices of the absent gap of $\sigma\left(P_{0}\right)$ and $\sigma\left(P_{1}\right)$, Yoshitomi [10] analyzed the discriminant defined as (2.4). However, we use the rotation number. In Section 4, we prove Theorem 1.2(a). For this purpose, we establish the modified Prüfer transform and use it to compute the rotation number. In Section 5, we give a proof of Theorem 1.2(b) by using the normal Prüfer transform and calculating the rotation number on $\bigcup_{k=1}^{\infty} B_{k} \cap B_{k+1}$.

\section{Location of the absent spectral gaps of $H$}

We henceforth suppose that (1.3) and $\kappa \neq \pi$ hold. Thanks to Proposition 1.1(e), we may assume, without any loss of generality, that

$$
A_{1} A_{2}=E .
$$

Since the $\delta$-interaction type was already analyzed in [10], we suppose that

$$
(a, b, d) \neq(1,0,1),(-1,0,-1),
$$

namely, we do not treat the case where $a=d$ and $b=0$. We now consider the equations

$$
\begin{gathered}
-y^{\prime \prime}(x, \lambda)=\lambda y(x, \lambda), \quad x \in \mathbb{R} \backslash \Gamma, \\
\left(\begin{array}{c}
y(x+0, \lambda) \\
y^{\prime}(x+0, \lambda)
\end{array}\right)=A_{j}\left(\begin{array}{c}
y(x-0, \lambda) \\
y^{\prime}(x-0, \lambda)
\end{array}\right), \quad x \in \Gamma_{j}, \quad j=1,2,
\end{gathered}
$$

where $\lambda$ is a real parameter. These equations have two solutions $y_{1}(x, \lambda)$ and $y_{2}(x, \lambda)$ which are uniquely determined by the initial conditions

$$
y_{1}(+0, \lambda)=1, \quad y_{1}^{\prime}(+0, \lambda)=0,
$$

and

$$
y_{2}(+0, \lambda)=0, \quad y_{2}^{\prime}(+0, \lambda)=1,
$$

respectively. We introduce the discriminant $D(\lambda)$ of (2.2) and (2.3):

$$
D(\lambda)=y_{1}(2 \pi+0, \lambda)+y_{2}^{\prime}(2 \pi+0, \lambda) .
$$

The matrix

$$
M(\lambda):=\left(\begin{array}{ll}
y_{1}(2 \pi+0, \lambda) & y_{2}(2 \pi+0, \lambda) \\
y_{1}^{\prime}(2 \pi+0, \lambda) & y_{2}^{\prime}(2 \pi+0, \lambda)
\end{array}\right)
$$

is called the monodromy matrix of (2.2) and (2.3). Since det $M(\lambda)=1$, the characteristic equation of $M(\lambda)$ is

$$
t^{2}-D(\lambda) t+1=0
$$

Thereby, $\lambda$ is an eigenvalue of $H_{\mu}$ if and only if $e^{i \mu}$ is a root of (2.6). Namely, the sequence $\left\{\lambda_{j}(\mu)\right\}_{j=1}^{\infty}$ provides all of the zeros of $D(\lambda)-2 \cos \mu$. By (1.4), (2.1), and a direct 
calculation, we obtain

$$
\begin{aligned}
y_{1}(2 \pi+0, \lambda)= & \cos \kappa \sqrt{\lambda} \cos \tau \sqrt{\lambda}-\left(\frac{a c}{\sqrt{\lambda}}+b d \sqrt{\lambda}\right) \cos \kappa \sqrt{\lambda} \sin \tau \sqrt{\lambda} \\
& -\left(a^{2}+b^{2} \lambda\right) \sin \kappa \sqrt{\lambda} \sin \tau \sqrt{\lambda} \\
y_{1}^{\prime}(2 \pi+0, \lambda)= & -\sqrt{\lambda} \sin \kappa \sqrt{\lambda} \cos \tau \sqrt{\lambda}-\left(\frac{c^{2}}{\sqrt{\lambda}}+d^{2} \sqrt{\lambda}\right) \cos \kappa \sqrt{\lambda} \sin \tau \sqrt{\lambda} \\
& -(a c+b d \lambda) \sin \kappa \sqrt{\lambda} \sin \tau \sqrt{\lambda}, \\
y_{2}(2 \pi+0, \lambda)= & \frac{1}{\sqrt{\lambda}} \sin \kappa \sqrt{\lambda} \cos \tau \sqrt{\lambda}+\left(\frac{a^{2}}{\sqrt{\lambda}}+b^{2} \sqrt{\lambda}\right) \cos \kappa \sqrt{\lambda} \sin \tau \sqrt{\lambda} \\
& -\left(\frac{a c}{\lambda}+b d\right) \sin \kappa \sqrt{\lambda} \sin \tau \sqrt{\lambda}, \\
y_{2}^{\prime}(2 \pi+0, \lambda)= & \cos \kappa \sqrt{\lambda} \cos \tau \sqrt{\lambda}+\left(\frac{a c}{\sqrt{\lambda}}+b d \sqrt{\lambda}\right) \cos \kappa \sqrt{\lambda} \sin \tau \sqrt{\lambda} \\
& -\left(\frac{c^{2}}{\lambda}+d^{2}\right) \sin \kappa \sqrt{\lambda} \sin \tau \sqrt{\lambda} .
\end{aligned}
$$

As described in [10, Lemma 4], $\lambda$ is a double eigenvalue of $H_{0}$ (respectively, $H_{\pi}$ ) if and only if $M(\lambda)=E$ (respectively, $M(\lambda)=-E$ ). Namely, we have

$$
\mathcal{B}:=\bigcup_{k=1}^{\infty} B_{k} \cap B_{k+1}=\{\lambda \in \mathbb{R} \mid M(\lambda)=E \text { or } M(\lambda)=-E\} .
$$

In this section, we show the following theorem.

THEOREM 2.1. We have the following.

(a) If $\kappa / \pi \notin \mathbb{Q}$, then we have

$$
\mathcal{B}= \begin{cases}\left\{\frac{k^{2}}{4}\right\} & \begin{array}{l}
\text { if } d=a, b \neq 0,-\frac{c}{b}=\frac{k^{2}}{4} \text { for some } k \in \mathbb{N}, \\
\text { otherwise. }
\end{array}\end{cases}
$$

(b) If $\kappa / 2 \pi=q / p,(p, q) \in \mathbb{N}^{2}$ and $\operatorname{gcd}(p, q)=1$, then we have

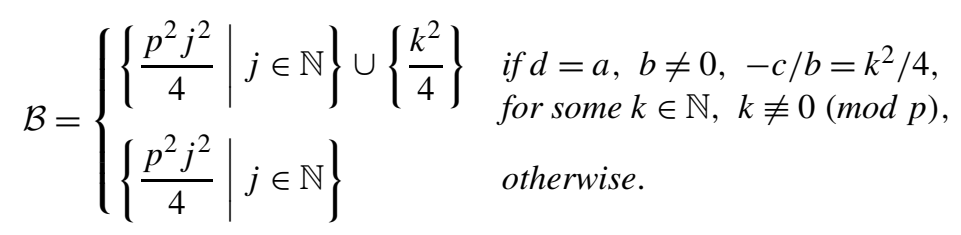

As the first step to establish Theorem 2.1, we prove the following lemma.

LemmA 2.2. We suppose that $M(\lambda)= \pm E$. Then we have $\lambda \neq 0$ and the following statements.

(i) If $d \neq \pm a$, then $\sin \kappa \sqrt{\lambda}=\sin \tau \sqrt{\lambda}=0$.

(ii) If $d=a, b \neq 0$, and $\lambda \neq-c / b$, then $\sin \kappa \sqrt{\lambda}=\sin \tau \sqrt{\lambda}=0$.

(iii) If $d=-a \neq 0$ and $\lambda \neq c / b$, then $\sin \kappa \sqrt{\lambda}=\sin \tau \sqrt{\lambda}=0$. 
Proof. We suppose that $M(\lambda)= \pm E$. We first show that $\lambda \neq 0$. Since

$$
M(0)=\left(\begin{array}{cc}
1-a c \tau & \kappa+a^{2} \tau-a c \kappa \tau \\
-c^{2} \tau & 1+a c \tau-c^{2} \kappa \tau
\end{array}\right),
$$

it follows that $M(0)= \pm E$ is equivalent to $c=0$ and $\kappa+a^{2} \tau=0$. By $\kappa>0$ and $\tau>0$, we have $\kappa+a^{2} \tau \neq 0$. Therefore, we have $\lambda \neq 0$.

Next we show that

$$
\frac{a c}{\sqrt{\lambda}}+d b \sqrt{\lambda}=0 \quad \text { and } \quad d^{2}=a^{2}
$$

if $\sin \kappa \sqrt{\lambda} \sin \tau \sqrt{\lambda} \neq 0$. Now we assume that

$$
\sin \kappa \sqrt{\lambda} \sin \tau \sqrt{\lambda} \neq 0 .
$$

We put $x_{1}=\cot \kappa \sqrt{\lambda}$ and $x_{2}=\cot \tau \sqrt{\lambda}$. Since $M(\lambda)= \pm E$, we have

$$
\begin{aligned}
& y_{1}(2 \pi+0, \lambda)-y_{2}^{\prime}(2 \pi+0, \lambda)=0, \\
& y_{2}(2 \pi+0, \lambda)=y_{1}^{\prime}(2 \pi+0, \lambda)=0 .
\end{aligned}
$$

Dividing the above equalities by $\sin \kappa \sqrt{\lambda} \sin \tau \sqrt{\lambda}$ and inserting (2.7)-(2.10) into them, we claim that

$$
\begin{gathered}
-2\left(\frac{a c}{\sqrt{\lambda}}+b d \sqrt{\lambda}\right) x_{1}+\left(d^{2}-a^{2}-b^{2} \lambda+\frac{c^{2}}{\lambda}\right)=0, \\
x_{2}+\left(\frac{c^{2}}{\lambda}+d^{2}\right) x_{1}+\left(\frac{a c}{\sqrt{\lambda}}+b d \sqrt{\lambda}\right)=0, \\
x_{2}+\left(a^{2}+b^{2} \lambda\right) x_{1}-\left(\frac{a c}{\sqrt{\lambda}}+b d \sqrt{\lambda}\right)=0 .
\end{gathered}
$$

Using (2.12)-(2.14), we obtain

$$
\left(\frac{a c}{\sqrt{\lambda}}+b d \sqrt{\lambda}\right)\left(x_{1}^{2}+1\right)=0 .
$$

Since $\cot z \neq \pm \sqrt{-1}$ for all $z \in \mathbf{C}$, we arrive at

$$
\frac{a c}{\sqrt{\lambda}}+b d \sqrt{\lambda}=0
$$

Substituting $a c / \sqrt{\lambda}+b d \sqrt{\lambda}=0, b d \lambda=-a c$ and $a c / \lambda=-b d$ for (2.12) multiplied by $a d$ and using $a d-b c=1$, we have $d^{2}=a^{2}$. So, (2.11) holds.

Next, we show that

$$
\sin \kappa \sqrt{\lambda}=\sin \tau \sqrt{\lambda}=0 \quad \text { if } \sin \kappa \sqrt{\lambda} \sin \tau \sqrt{\lambda}=0 .
$$

If $\sin \tau \sqrt{\lambda}=0$, then it follows by $0=y_{1}^{\prime}(2 \pi+0, \lambda)=-\sqrt{\lambda} \sin \kappa \sqrt{\lambda} \cos \tau \sqrt{\lambda}$ and $\lambda \neq 0$ that $\sin \kappa \sqrt{\lambda}=0$. Namely, we have

$$
\sin \kappa \sqrt{\lambda}=0 \text { if } \sin \tau \sqrt{\lambda}=0 .
$$

Next, we prove the converse:

$$
\sin \tau \sqrt{\lambda}=0 \quad \text { if } \sin \kappa \sqrt{\lambda}=0
$$


by contradiction. Seeking a contradiction, we now assume that

$$
\sin \kappa \sqrt{\lambda}=0 \quad \text { and } \quad \sin \tau \sqrt{\lambda} \neq 0 .
$$

Then it follows from $y_{1}(2 \pi+0, \lambda)-y_{2}^{\prime}(2 \pi+0, \lambda)=0$ and $y_{1}^{\prime}(2 \pi+0, \lambda)=y_{2}(2 \pi+$ $0, \lambda)=0$ that

$$
a c+b d \lambda=c^{2}+d^{2} \lambda=a^{2}+b^{2} \lambda=0 .
$$

Multiplying $a c+b d \lambda=0$ by $a d$ and then substituting $d^{2} \lambda=-c^{2}$ for its result, we have $a c=0$. It follows by $c^{2}+d^{2} \lambda=a^{2}+b^{2} \lambda=0, \lambda \neq 0$, and $a c=0$ that

$$
(a, b)=(0,0) \quad \text { or } \quad(c, d)=(0,0)
$$

In both cases, these contradict $a d-b c=1$. Therefore, we obtain (2.17). Combining (2.16) and (2.17), we have (2.15).

Finally, we show (i)-(iii) by using (2.11) and (2.15). The statement (i) directly follows from (2.11) and (2.15). We prove (ii). We suppose that $d=a, b \neq 0$ and $\lambda \neq-c / b$. Since $\lambda$ does not satisfy $a c / \sqrt{\lambda}+b d \sqrt{\lambda}=0$ if $d=a \neq 0$, we have $\sin \kappa \sqrt{\lambda}=\sin \tau \sqrt{\lambda}=0$ if $d=a \neq 0$. We show that $\sin \kappa \sqrt{\lambda}=\sin \tau \sqrt{\lambda}=0$ if $d=a=0$. We assume that $d=a=0$. Since $c=-1 / b, \lambda \neq-c / b$ implies $\lambda \neq 1 / b^{2}$. Substituting $d=a=0$ for $y_{1}(2 \pi+0, \lambda)-$ $y_{2}^{\prime}(2 \pi+0, \lambda)=0$, we have

$$
\left(b^{2} \lambda-\frac{1}{b^{2} \lambda}\right) \sin \kappa \sqrt{\lambda} \sin \tau \sqrt{\lambda}=0 .
$$

This combined with $\lambda \neq 1 / b^{2}$ implies

$$
\lambda=-1 / b^{2} \quad \text { or } \quad \sin \kappa \sqrt{\lambda} \sin \tau \sqrt{\lambda}=0 .
$$

So, we have only to prove $\lambda \neq-1 / b^{2}$ because of (2.15). Since

$$
M\left(-\frac{1}{b^{2}}\right)=\left(\begin{array}{cc}
\cosh \left(\frac{\kappa-\tau}{|b|}\right) & |b| \sinh \left(\frac{\kappa-\tau}{|b|}\right) \\
\frac{1}{|b|} \sinh \left(\frac{\kappa-\tau}{|b|}\right) & \cosh \left(\frac{\kappa-\tau}{|b|}\right)
\end{array}\right) \neq \pm E
$$

we observe that $\lambda \neq-1 / b^{2}$. Therefore, we conclude (ii). Our last work is to prove (iii). In order to show (iii), we assume that $d=-a \neq 0$ and $\lambda \neq c / b$. Since $\lambda$ does not satisfy $a c / \sqrt{\lambda}+b d \sqrt{\lambda}=0$, it follows by (2.11) and (2.15) that (iii) is valid.

Next, we complement Lemma 2.2 in the following lemma.

LEMMA 2.3. We have the following statements.

(i) If $d=-a \neq 0$, then we have $M(c / b) \neq \pm E$.

(ii) Suppose that $d=a$ and $b \neq 0$. Then, the following two statements (a) and (b) are equivalent:

(a) there exists $k \in \mathbb{N}$ such that $-c / b=k^{2} / 4$;

(b) $M(-c / b)= \pm E$. 
Proof. First, we show (i) by contradiction. We assume $d=-a \neq 0$. Seeking a contradiction, we suppose that $M(c / b)= \pm E$. We define

$$
\operatorname{sgn}(x)= \begin{cases}1 & \text { if } x>0 \\ 0 & \text { if } x=0 \\ -1 & \text { if } x<0\end{cases}
$$

We note that

$$
\lambda:=\frac{c}{b}=\left|\frac{c}{b}\right| \operatorname{sgn}\left(\frac{c}{b}\right) .
$$

Since $a=-d \neq 0$, we obtain $-a^{2}-b c=1$. Thereby, we derive

$$
\operatorname{sgn}\left(\frac{c}{b}\right)=\operatorname{sgn}(b c)=\operatorname{sgn}\left(-a^{2}-1\right)<0,
$$

from which

$$
\sqrt{\lambda}=\sqrt{|c / b|} \sqrt{-1} .
$$

This together with $y_{1}^{\prime}(2 \pi+0, c / b)=0$ implies that

$$
0=\sin (\kappa-\tau) \sqrt{\lambda}=\sinh (\kappa-\tau) \sqrt{|c / b|} .
$$

Since $c \neq 0$ by Lemma 2.2, we have $\kappa-\tau=0$, namely, $\kappa=\pi$ which is a contradiction. Therefore, we obtain (i).

Next, we show (ii). We suppose that (b) holds. By Lemma 2.2, we have $c \neq 0$. Since $y_{2}(2 \pi+0,-c / b)=0, d=a$ and $a^{2}-b c=1$, we obtain $\sin 2 \pi \sqrt{-c / b}=0$. This combined with $c \neq 0$ implies (a). Conversely, we suppose that (a) is valid. Then, it follows by $a=d$ and $a^{2}-b c=1$ that

$$
a=d= \pm \sqrt{1-\frac{k^{2}}{4} b^{2}} .
$$

By a straightforward computation, we have $M(-c / b)=(-1)^{k} E$, i.e. (b) is valid. Therefore, we conclude that (ii) holds.

Summarizing Lemmas 2.2 and 2.3, we arrive at the following assertion.

LEMMA 2.4. We have the following.

(i) If $d \neq \pm a$, then we have

$$
\mathcal{B}=\{\lambda \in \mathbb{R} \backslash\{0\} \mid \sin \kappa \sqrt{\lambda}=\sin \tau \sqrt{\lambda}=0\} .
$$

(ii) If $d=-a \neq 0$, then we have

$$
\mathcal{B}=\{\lambda \in \mathbb{R} \backslash\{0, c / b\} \mid \sin \kappa \sqrt{\lambda}=\sin \tau \sqrt{\lambda}=0\} .
$$

(iii) Assume that $d=a$ and $b \neq 0$. Then we have the following two statements.

(1) If $-c / b \notin\left\{k^{2} / 4 \mid k \in \mathbb{N}\right\}$, then we have

$$
\mathcal{B}=\{\lambda \in \mathbb{R} \backslash\{0,-c / b\} \mid \sin \kappa \sqrt{\lambda}=\sin \tau \sqrt{\lambda}=0\} .
$$


(2) If $-c / b=k^{2} / 4$ and $k \in \mathbb{N}$, then we have

$$
\mathcal{B}=\left\{\lambda \in \mathbb{R} \backslash\left\{0, \frac{k^{2}}{4}\right\} \mid \sin \kappa \sqrt{\lambda}=\sin \tau \sqrt{\lambda}=0\right\} \cup\left\{\frac{k^{2}}{4}\right\} .
$$

Proof. We prove (ii). By Lemmas 2.2 and 2.3, we have

$$
\mathcal{B} \subset\{\lambda \in \mathbb{R} \backslash\{0, c / b\} \mid \sin \kappa \sqrt{\lambda}=\sin \tau \sqrt{\lambda}=0\} .
$$

Conversely, we pick $\lambda_{0} \in\{\lambda \in \mathbb{R} \backslash\{0, c / b\} \mid \sin \kappa \sqrt{\lambda}=\sin \tau \sqrt{\lambda}=0\}$, arbitrarily. Since $\sin \kappa \sqrt{\lambda_{0}}=\sin \tau \sqrt{\lambda_{0}}=0$, it follows by (2.7)-(2.10) that $M\left(\lambda_{0}\right)= \pm E$. This implies

$$
\mathcal{B} \supset\{\lambda \in \mathbb{R} \backslash\{0, c / b\} \mid \sin \kappa \sqrt{\lambda}=\sin \tau \sqrt{\lambda}=0\} .
$$

Therefore, we obtain (ii). By a similar way, we obtain (i) and (iii).

Owing to Lemma 2.4 , the set $\mathcal{B}$ is rather simplified. It is time to bring Theorem 2.1 to completion.

Proof of Theorem 2.1. Since $\{z \in \mathbf{C} \mid \sin z=0\}=\pi \mathbb{Z}$ and $\tau=2 \pi-\kappa$, the following two statements are equivalent:

- there exists $\lambda \neq 0$ such that $\sin \kappa \sqrt{\lambda}=\sin \tau \sqrt{\lambda}=0$;

- $\kappa \in \pi \mathbb{Q}$.

We first prove (a). Let $\kappa / \pi \notin \mathbb{Q}$. As described above, we obtain

$$
\{\lambda \in \mathbb{R} \backslash\{0\} \mid \sin \kappa \sqrt{\lambda}=\sin \tau \sqrt{\lambda}=0\}=\emptyset .
$$

This together with Lemma 2.4 yields (a).

Next, let us show (b). Put $\kappa / 2 \pi=q / p,(p, q) \in \mathbb{N}^{2}$ and $\operatorname{gcd}(p, q)=1$. Then, we have

$$
\{\lambda \in \mathbb{R} \backslash\{0\} \mid \sin \kappa \sqrt{\lambda}=\sin \tau \sqrt{\lambda}=0\}=\left\{\frac{p^{2} j^{2}}{4} \mid j \in \mathbb{N}\right\} .
$$

Combining this with Lemma 2.4(i), we obtain (b) for $d \neq \pm a$. Next, we suppose that $d=-a \neq 0$. Since $b c=-a^{2}-1<0$, we have $c / b \notin\left\{p^{2} j^{2} / 4 \mid j \in \mathbb{N}\right\}$. Therefore, we obtain

$$
\mathcal{B}=\{\lambda \in \mathbb{R} \backslash\{0, c / b\} \mid \sin \kappa \sqrt{\lambda}=\sin \tau \sqrt{\lambda}=0\}=\left\{\frac{p^{2} j^{2}}{4} \mid j \in \mathbb{N}\right\} .
$$

So, we have (b) for $d=-a \neq 0$. Finally we show (b) in the case where $d=a$ and $b \neq 0$. Suppose that $-c / b \notin\left\{k^{2} / 4 \mid k \in \mathbb{N}\right\}$. Then, it follows by Lemma 2.4(iii)(1) that

$$
\begin{aligned}
\mathcal{B} & =\{\lambda \in \mathbb{R} \backslash\{0,-c / b\} \mid \sin \kappa \sqrt{\lambda}=\sin \tau \sqrt{\lambda}=0\} \\
& =\left\{\frac{p^{2} j^{2}}{4} \mid j \in \mathbb{N}\right\} \backslash\left\{-\frac{c}{b}\right\} \\
& =\left\{\frac{p^{2} j^{2}}{4} \mid j \in \mathbb{N}\right\} .
\end{aligned}
$$


On the other hand, we suppose that $-c / b=k^{2} / 4$ and $k \in \mathbb{N}$. Then, it follows by Lemma 2.4(iii)(2) that

$$
\begin{aligned}
\mathcal{B} & =\left\{\lambda \in \mathbb{R} \backslash\left\{0, \frac{k^{2}}{4}\right\} \mid \sin \kappa \sqrt{\lambda}=\sin \tau \sqrt{\lambda}=0\right\} \cup\left\{\frac{k^{2}}{4}\right\} \\
& =\left(\left\{\frac{p^{2} j^{2}}{4} \mid j \in \mathbb{N}\right\} \backslash\left\{\frac{k^{2}}{4}\right\}\right) \cup\left\{\frac{k^{2}}{4}\right\} \\
& =\left\{\begin{array}{lll}
\left\{\frac{p^{2} j^{2}}{4} \mid j \in \mathbb{N}\right\} & \text { if } k \equiv 0 \quad(\bmod p), \\
\left\{\frac{p^{2} j^{2}}{4} \mid j \in \mathbb{N}\right\} \cup\left\{\frac{k^{2}}{4}\right\} & \text { if } k \neq 0 \quad(\bmod p) .
\end{array}\right.
\end{aligned}
$$

Thus, we obtain (b) for $d=a$ and $b \neq 0$.

\section{The rotation number}

In this section, we introduce the rotation number for the generalized Kronig-Penney Hamiltonians. We then use it to prove Theorem 1.2 in Sections 4 and 5. To recall the definition of the rotation number, we consider the Schrödinger equations (2.2) and (2.3). Let $y$ be a nontrivial solution of (2.2) and (2.3). By $(r, \omega)$, we denote the polar coordinates of $\left(y, y^{\prime}\right)$ :

$$
y=r \sin \omega, \quad y^{\prime}=r \cos \omega .
$$

Then the function $\omega=\omega(x, \lambda)$ is called the Prüfer transform of $y(x, \lambda)$. The function $\omega(x, \lambda)$ satisfies the equation

$$
\frac{d}{d x} \omega(x, \lambda)=\cos ^{2} \omega(x, \lambda)+\lambda \sin ^{2} \omega(x, \lambda), \quad x \in \mathbb{R} \backslash \Gamma
$$

as well as the boundary conditions

$$
\begin{gathered}
\sin \omega(x+0, \lambda)\left(c_{j} \sin \omega(x-0, \lambda)+d_{j} \cos \omega(x-0, \lambda)\right) \\
=\cos \omega(x+0, \lambda)\left(a_{j} \sin \omega(x-0, \lambda)+b_{j} \cos \omega(x-0, \lambda)\right), \\
\operatorname{sgn}(\sin \omega(x+0, \lambda))=\operatorname{sgn}\left(a_{j} \sin \omega(x-0, \lambda)+b_{j} \cos \omega(x-0, \lambda)\right), \\
\operatorname{sgn}(\cos \omega(x+0, \lambda))=\operatorname{sgn}\left(c_{j} \sin \omega(x-0, \lambda)+d_{j} \cos \omega(x-0, \lambda)\right)
\end{gathered}
$$

for all $x \in \Gamma_{j}$. Following [7, Theorem 1.2], we choose the branch of $\omega(x+0, \lambda)$ as

$$
\omega(x+0, \lambda)-\omega(x-0, \lambda) \in[-\pi, \pi) \text { for } x \in \Gamma .
$$

Let $\omega=\omega\left(x, \lambda, \omega_{0}\right)$ be the solution of (3.2)-(3.6) subject to the initial condition

$$
\omega(+0, \lambda)=\omega_{0} \in \mathbb{R} .
$$

We define the rotation number of (3.2)-(3.6) as

$$
\rho(\lambda)=\lim _{n \rightarrow \infty} \frac{\omega\left(2 n \pi+0, \lambda, \omega_{0}\right)-\omega_{0}}{2 n \pi} .
$$

We quote [7, Theorem 1.2], which relates $\rho(\lambda)$ to the spectrum of $H$. 
THEOREM A. For $A_{1}, A_{2} \in \mathrm{SL}_{2}(\mathbb{R})$ and $\kappa \in(0,2 \pi)$, we have the following.

(a) The limit on the right-hand side of (3.8) exists and is independent of the initial value $\omega_{0}$.

(b) The function $\rho(\lambda)$ is non-decreasing on $\mathbb{R}$.

(c) We put

$$
B_{m}=\left[\alpha_{m}, \beta_{m}\right] \quad \text { for } m \in \mathbb{N}
$$

and

$$
l=\sharp\left\{j \in\{1,2\} \mid\left(b_{j}<0\right) \text { or }\left(b_{j}=0, d_{j}<0\right)\right\},
$$

where $\sharp A$ stands for the number of the elements of $A$ for a finite set $A$. Then, for $m \in \mathbb{N}$, we have

$$
\begin{gathered}
\alpha_{m}=\max \left\{\lambda \in \mathbb{R} \mid \rho(\lambda)=\frac{m-1}{2}-\frac{l}{2}\right\}, \\
\beta_{m}=\min \left\{\lambda \in \mathbb{R} \mid \rho(\lambda)=\frac{m}{2}-\frac{l}{2}\right\}
\end{gathered}
$$

\section{Proof of Theorem 1.2 for $\kappa \notin \pi \mathbb{Q}$}

In this section, we suppose that $\kappa \notin \pi \mathbb{Q}$. Owing to Theorem 2.1 and [10, Theorem 3(i)], we have $\Lambda \neq \emptyset$ if and only if

$$
d=a, \quad b \neq 0 \quad \text { and } \quad-\frac{c}{b}=\frac{k^{2}}{4} \quad \text { for some } k \in \mathbb{N} .
$$

So, it suffices to consider the case (4.1). By (4.1) and $a d-b c=1$, we have $a^{2}+k^{2} b^{2} / 4=1$. This implies that there exists $\theta \in(-\pi, 0) \cup(0, \pi)$ such that

$$
a=\cos \theta \quad \text { and } \quad b=\frac{2}{k} \sin \theta .
$$

We newly introduce the modified Prüfer transform, which is an effective tool for our purpose. Substituting (4.1) and (4.2) for (2.3), we can rewrite the boundary conditions on $\Gamma$ as

$$
\begin{aligned}
& \left(\begin{array}{c}
y(x+0, \lambda) \\
y^{\prime}(x+0, \lambda)
\end{array}\right)=\left(\begin{array}{cc}
\cos \theta & \frac{2}{k} \sin \theta \\
-\frac{k}{2} \sin \theta & \cos \theta
\end{array}\right)\left(\begin{array}{c}
y(x-0, \lambda) \\
y^{\prime}(x-0, \lambda)
\end{array}\right) \quad \text { for } x \in \Gamma_{1}, \\
& \left(\begin{array}{c}
y(x+0, \lambda) \\
y^{\prime}(x+0, \lambda)
\end{array}\right)=\left(\begin{array}{cc}
\cos \theta & -\frac{2}{k} \sin \theta \\
\frac{k}{2} \sin \theta & \cos \theta
\end{array}\right)\left(\begin{array}{c}
y(x-0, \lambda) \\
y^{\prime}(x-0, \lambda)
\end{array}\right) \quad \text { for } x \in \Gamma_{2} .
\end{aligned}
$$

Let $(\tilde{r}, \tilde{\omega})$ stand for the elliptic polar coordinates of $\left(y, y^{\prime}\right)$ :

$$
\begin{aligned}
& y(x, \lambda)=\frac{2}{k} \tilde{r}(x, \lambda) \sin \tilde{\omega}(x, \lambda), \\
& y^{\prime}(x, \lambda)=\tilde{r}(x, \lambda) \cos \tilde{\omega}(x, \lambda) .
\end{aligned}
$$


We call the function $\tilde{\omega}(x, \lambda)$ the modified Prüfer transform of $y(x, \lambda)$. The function $\tilde{\omega}(x, \lambda)$ satisfies the equations

$$
\begin{gathered}
\frac{d}{d x} \tilde{\omega}(x, \lambda)=\frac{k}{2} \cos ^{2} \tilde{\omega}(x, \lambda)+\frac{2}{k} \lambda \sin ^{2} \tilde{\omega}(x, \lambda), \quad x \in \mathbb{R} \backslash \Gamma, \\
\tilde{\omega}(x+0, \lambda)=\tilde{\omega}(x-0, \lambda)+\theta, \quad x \in \Gamma_{1}, \\
\tilde{\omega}(x+0, \lambda)=\tilde{\omega}(x-0, \lambda)-\theta, \quad x \in \Gamma_{2} .
\end{gathered}
$$

Let $\tilde{\omega}=\tilde{\omega}\left(x, \lambda, \omega_{0}\right)$ be the solution of (4.7)-(4.9) subject to the initial condition

$$
\tilde{\omega}(+0, \lambda)=\omega_{0}
$$

Since $|\tilde{\omega}(x, \lambda, 0)-\omega(x, \lambda, 0)|<\pi / 2$, we have

$$
\begin{aligned}
& \left|\rho(\lambda)-\frac{\tilde{\omega}(2 n \pi+0, \lambda, 0)}{2 n \pi}\right| \\
& \quad \leq\left|\rho(\lambda)-\frac{\omega(2 n \pi+0, \lambda, 0)}{2 n \pi}\right|+\left|\frac{\omega(2 n \pi+0, \lambda, 0)-\tilde{\omega}(2 n \pi+0, \lambda, 0)}{2 n \pi}\right| \\
& \quad \rightarrow 0
\end{aligned}
$$

as $n \rightarrow \infty$, namely,

$$
\rho(\lambda)=\lim _{n \rightarrow \infty} \frac{\tilde{\omega}(2 n \pi+0, \lambda, 0)}{2 n \pi} .
$$

By using the modified Prüfer transform, we prove Theorem 1.2(a).

Proof of Theorem 1.2(a). Since $\mathcal{B}=\left\{k^{2} / 4\right\}$ by Theorem 2.1 , we have only to calculate $\rho\left(k^{2} / 4\right)$. Substituting $\lambda=k^{2} / 4$ for (4.7), we have a simple equation

$$
\frac{d}{d x} \tilde{\omega}\left(x, \frac{k^{2}}{4}\right)=\frac{k}{2} \quad \text { for } x \in \mathbb{R} \backslash \Gamma .
$$

Since the rotation number is independent of the initial value $\omega_{0}$, we may put $\omega_{0}=0$. We have, of course,

$$
\tilde{\omega}\left(x, \frac{k^{2}}{4}, 0\right)= \begin{cases}\frac{k}{2} x, & x \in(0, \kappa)+2 \pi \mathbb{Z}, \\ \frac{k}{2} x-\theta, & x \in(\kappa, 2 \pi)+2 \pi \mathbb{Z} .\end{cases}
$$

This ease is why we have introduced the modified Prüfer transform, instead of the normal one. Formula (4.11) implies $\tilde{\omega}\left(2 \pi t+0, k^{2} / 4\right)=k \pi t$ for $t \in \mathbb{N}$, from which $\rho\left(k^{2} / 4\right)=k / 2$. Combining this with Theorem A, we conclude that the $(k+1)$ th spectral gap is absent. Therefore, we obtain Theorem 1.2 for $\kappa \notin \pi \mathbb{Q}$.

Remark 4.1. By the above argument, we also infer that if (4.1) and $\kappa \in \pi \mathbb{Q}$ are fulfilled, then the $(k+1)$ th gap of $\sigma(H)$ is absent.

Remark 4.2. When $k=2$, we have $A_{1}, A_{2} \in \mathrm{SO}(2) \backslash\{E,-E\}$ and $A_{1} A_{2} \in\{E,-E\}$. This case has been investigated in [6, Theorem 1.3]. 


\section{Proof of Theorem 1.2 for $\kappa \in \pi \mathbb{Q}$}

The aim of this section is to determine all of the indices of the absent spectral gaps for $\kappa \in \pi \mathbb{Q}$. Let $\kappa / 2 \pi=q / p,(p, q) \in \mathbb{N}^{2}$, and $\operatorname{gcd}(p, q)=1$. To prove Theorem 1.2(a), we use Theorem A and the normal Prüfer transform.

We first deal with the case where $d=a$ and $b \neq 0$.

LEMMA 5.1. Suppose that $d=a$ and $b \neq 0$. Then we have

$$
\Lambda \supset\{1+p j \mid j \in \mathbb{N}\} .
$$

Proof. First, we shall calculate $\rho\left(p^{2} j^{2} / 4\right)$. For $j \in \mathbb{N}$, we put $\lambda_{j}=p^{2} j^{2} / 4$. Since $\rho(\lambda)$ is independent of the initial value $\omega_{0}$, we may put $\omega_{0}=0$. The corresponding solution to $\omega(x, \lambda, 0)$ is $y_{2}(x, \lambda)$. We have

$$
y_{2}\left(x, \lambda_{j}\right)=\frac{2}{p j} \sin \frac{p j}{2} x \quad \text { and } \quad y_{2}^{\prime}\left(x, \lambda_{j}\right)=\cos \frac{p j}{2} x
$$

for $x \in(0, \kappa)$. This combined with $\kappa / 2 \pi=q / p$ implies

$$
\omega\left(\kappa-0, \lambda_{j}, 0\right)=q j \pi .
$$

Substituting this for (3.3)-(3.6), we have

$$
\begin{gathered}
a \sin \omega\left(\kappa+0, \lambda_{j}, 0\right)=-b \cos \omega\left(\kappa+0, \lambda_{j}, 0\right), \\
\operatorname{sgn}\left(\sin \omega\left(\kappa+0, \lambda_{j}, 0\right)\right)=-(-1)^{q j} \operatorname{sgn}(b), \\
\operatorname{sgn}\left(\cos \omega\left(\kappa+0, \lambda_{j}, 0\right)\right)=(-1)^{q j} \operatorname{sgn}(a), \\
\omega\left(\kappa+0, \lambda_{j}, 0\right) \in[(q j-1) \pi,(q j+1) \pi) .
\end{gathered}
$$

Using these four equations, we obtain

$$
\omega\left(\kappa+0, \lambda_{j}, 0\right)= \begin{cases}q \pi j-\pi / 2 & \text { if } a=0, b>0, \\ q \pi j+\pi / 2 & \text { if } a=0, b<0, \\ q \pi j-\operatorname{Arctan}(b / a) & \text { if } a>0, b>0, \\ q \pi j-\operatorname{Arctan}(b / a) & \text { if } a>0, b<0, \\ q \pi j-\operatorname{Arctan}(b / a)+\pi & \text { if } a<0, b<0, \\ q \pi j-\operatorname{Arctan}(b / a)-\pi & \text { if } a<0, b>0 .\end{cases}
$$

We have

$$
y_{2}\left(x, \lambda_{j}\right)=(-1)^{q j}\left(\frac{2 a}{p j} \sin \frac{p j}{2}(x-\kappa)-b \cos \frac{p j}{2}(x-\kappa)\right),
$$

and

$$
y_{2}^{\prime}\left(x, \lambda_{j}\right)=(-1)^{q j}\left(a \cos \frac{p j}{2}(x-\kappa)+\frac{p j b}{2} \sin \frac{p j}{2}(x-\kappa)\right)
$$

for $x \in(\kappa, 2 \pi)$. As in the above discussion, we obtain

$$
\omega\left(2 \pi+0, \lambda_{j}, 0\right)=p \pi j .
$$


Since (3.2) is $\pi$-periodic in $\omega$, by (5.1) we have

$$
\omega\left(2 \pi t+0, \lambda_{j}, 0\right)=p \pi j t
$$

for $t \in \mathbb{N}$. Therefore, we arrive at

$$
\rho\left(\frac{p^{2} j^{2}}{4}\right)=\frac{p j}{2} .
$$

We recall (3.9). Since $d=a$ and $b \neq 0$ by the assumption, we note that $l=1$. By Theorem A, we have the desired statement.

By Theorem 2.1, Remark 4.1, and Lemma 5.1, we obtain Theorem 1.2(b) for $d=a$ and $b \neq 0$. On the other hand, [10, Theorem 3(ii) and (iii)] directly means Theorem 1.2(b) for $d=a$ and $b=0$.

Next, we show Theorem 1.2(b) for $d=-a \neq 0$. Suppose that $d=-a \neq 0$. We recall Theorem 2.1(b). We pick $j \in \mathbb{N}$, arbitrarily. Then, we obtain

$$
\rho\left(\frac{p^{2} j^{2}}{4}\right)=\frac{p j}{2} \quad \text { and } \quad l=1
$$

in a similar way to Lemma 5.1. Therefore, we obtain the statement of Theorem 2.1(b) for $d=-a \neq 0$.

Finally, we prove Theorem 2.1(b) in the case where $d \neq \pm a$. A similar argument to the proof of Lemma 5.1 gives

$$
\rho\left(\frac{p^{2} j^{2}}{4}\right)= \begin{cases}\frac{p j-2}{2} & \text { if } b=0, a<0, \\ \frac{p j}{2} & \text { otherwise, }\end{cases}
$$

and

$$
l= \begin{cases}0 & \text { if } b=0, a>0 \\ 2 & \text { if } b=0, a<0 \\ 1 & \text { otherwise } .\end{cases}
$$

Therefore, these combined with Theorem A yield Theorem 1.2(b) for $d \neq \pm a$.

Acknowledgements. The author thanks the referee for useful comments which improved the manuscript. He is also grateful for Professor Kazushi Yoshitomi for helpful advice.

\section{REFERENCES}

[1] P. R. Chernoff and R. J. Hughes. A new class of point interactions in one dimension. J. Funct. Anal. 111 (1993), 97-117.

[2] F. Gesztesy, H. Holden and W. Kirsch. On energy gaps in a new type of analytically solvable model in quantum mechanics. J. Math. Anal. Appl. 134 (1988), 9-29.

[3] F. Gesztesy and W. Kirsch. One-dimensional Schrödinger operators with interactions singular on a discrete set. J. reine angew. Math. 362 (1985), 28-50.

[4] R. J. Hughes. Generalized Kronig-Penney Hamiltonians. J. Math. Anal. Appl. 222(1) (1998), 151-166.

[5] R. Kronig and W. Penney. Quantum mechanics in crystal lattices. Proc. R. Soc. London 130 (1931), 499-513. 
[6] H. Niikuni. Identification of the absent spectral gaps in a class of generalized Kronig-Penney Hamiltonians. Tsukuba J. Math. 31(1) (2007), 39-65.

[7] H. Niikuni. The rotation number for the generalized Kronig-Penney Hamiltonians. Ann. H. Poincaré. 8 (2007), 1279-1301.

[8] M. Reed and B. Simon. Analysis of Operators (Methods of Modern Mathematical Physics, IV). Academic Press, New York, 1978.

[9] P. Šeba. The generalized point interaction in one dimension. Czech J. Phys. B 36 (1986), 667-673.

[10] K. Yoshitomi. Spectral gaps of the one-dimensional Schrödinger operators with periodic point interactions. Hokkaido Math. J. 35(2) (2006), 365-378.

\author{
Hiroaki Niikuni \\ Department of Mathematics and Information Sciences \\ Tokyo Metropolitan University \\ 1-1 Minami-Ohsawa \\ Hachioji, 192-0397 Tokyo, Japan \\ (E-mail:dreamsphere@infoseek.jp)
}

